\title{
Hyperuricemia is Associated With an Increased Prevalence of Metabolic Syndrome in a General Population and a Decreased Prevalence of Diabetes in Men
}

\author{
Hyemin Jeong, M.D., Ph.D. ${ }^{1}$, Ji Eun Moon, Ph.D. ${ }^{2}$, Chan Hong Jeon, M.D., Ph.D. ${ }^{1}$ \\ ${ }^{1}$ Division of Rheumatology, Department of Internal Medicine, ${ }^{2}$ Department of Biostatistics, Soonchunhyang University Hospital Bucheon, \\ Bucheon, Korea
}

\begin{abstract}
Objective. Elevated uric acid is associated with cardiovascular disease and metabolic syndrome. However, uric acid is also an antioxidant with beneficial effect on comorbidities. The aim of this study was to evaluate the relationship of serum uric acid with diabetes, metabolic syndrome, and cardiovascular disease in a Korean adult population. Methods. A total of 5,887 (weighted $\mathrm{n}=40,251,868$ ) participants aged $\geq 19$ years from the 2016 Korean National Health and Nutrition Examination Survey were included for analysis. Weighted prevalence and odds ratio (OR) of comorbidities were analyzed according to the presence of hyperuricemia and uric acid quartile. Results. Participants of both sexes with hyperuricemia showed higher prevalence of metabolic syndrome, hypertension, hypertriglyceridemia, and obesity than those without hyperuricemia. After adjusting for socioeconomic and lifestyle characteristics, hyperuricemia was associated with a decreased prevalence of diabetes mellitus in men (OR: $0.44,95 \%$ confidence interval [Cl]: $0.28 \sim 0.72, p=0.001$ ) and a decreased prevalence of myocardial infarction or angina (OR: $0.25,95 \% \mathrm{Cl}: 0.08 \sim 0.75, \mathrm{p}=0.013$ ) in women. Hyperuricemia was significantly associated with an increased prevalence of metabolic syndrome in both men (OR: 1.81, 95\% Cl: 1.33 2.45, p<0.001) and women (OR: 1.95, 95\% Cl: 1.22 $3.13, p=0.006)$. Conclusion. Hyperuricemia was associated with a decreased prevalence of diabetes mellitus in men and a decreased prevalence of myocardial infarction or angina in women. Hyperuricemia was associated with an increased prevalence of metabolic syndrome in both men and women. (J Rheum Dis 2020;27:247-260)
\end{abstract}

Key Words. Uric acid, Metabolic syndrome, Diabetes mellitus

\section{INTRODUCTION}

Uric acid, the final oxidation product of purine metabolism in humans and higher primates, is influenced by genetics, diet, alcohol consumption, and renal insufficiency. Hyperuricemia is involved in the pathogenesis of gout. Excess serum uric acid concentrations are associated with comorbidities such as coronary artery disease, stroke, metabolic syndrome, diabetes, and hypertension [1-4]. Uric acid can induce endothelial dysfunction [5] and contribute to systemic inflammation [6]. It is also a prooxidant and a marker of oxidative stress [7]. Fructose-induced uric acid generation can cause mitochondrial oxi- dative stress and result in insulin resistance [8]. These might be potential mechanisms involved in the role of uric acid as a cardiovascular risk factor.

However, serum uric acid concentrations are positively associated with longevity in humans and mammals [9], implying that uric acid might have an physiologic role in the human body. Uric acid is an endogenous antioxidant that provides an antioxidant defense in humans against oxidant-and radical-caused diseases [10]. Extremely low serum uric acid concentrations have been associated with endothelial dysfunction and adverse cardiovascular events [11]. Uric acid administration can restore endothelial function of patients with diabetes and smokers [12]. Uric

Received : April 1, 2020, Revised : (1st) June 18, 2020, (2nd) August 18, 2020, Accepted : August 19, 2020

Corresponding to : Chan Hong Jeon (Dhttp://orcid.org/0000-0002-2430-7264

Division of Rheumatology, Department of Internal Medicine, Soonchunhyang University Hospital Bucheon, 170 Jomaru-ro, Wonmi-gu, Bucheon 14584, Korea. E-mail : chjeon@gmail.com

Copyright (c) 2020 by The Korean College of Rheumatology.

This is an Open Access article, which permits unrestricted non-commerical use, distribution, and reproduction in any medium, provided the original work is properly cited. 
acid levels are inversely associated with diabetes in a US population [13]. On the other hand, serum uric acid is negatively associated with diabetes in Japanese men, but not in women [14]. Serum uric acid levels are associated with severe coronary artery disease only in women [15]. The relationship between uric acid and cardiovascular risk is still controversial. Therefore, the aim of this study was to evaluate the relationship of serum uric acid with diabetes, metabolic syndrome, and cardiovascular disease in men and women Korean adults.

\section{MATERIALS AND METHODS}

\section{Study population}

The Korean National Health and Nutrition Examination Survey (KNHANES) is a nationwide survey that is conducted periodically by the Korean Centers for Disease Control and Prevention to investigate the health and nutritional status of the Korean population [16]. This survey assesses the general health and nutritional status of individuals in South Korea through interviews regarding health and nutrition and basic health assessments. Study participants were selected using a proportional allocation-systematic sampling method with multistage stratification to derive a representative Korean population. Although individual participants were not generally representatives of the Korean population, this survey provided representative estimates of the noninstitutionalized Korean civilian population using the power of sample weight. Every year, 10,000 to 12,000 individuals in approximately 3,800 to 4,600 households are selected from a panel based on National Census Data. Participation rates of selected households in the past several cycles of the KNHANES have been high, ranging from $75 \%$ to $80 \%$. This study analyzed the 2016 KNHANES [17]. In the 2016 KNANES, 10,806 individuals in 4,416 households were selected. Participation rate was $75.4 \%$. Among 8,150 participants in the 2016 KNHANES, 1,768 participants who were younger than 19 years old were excluded. Among 6,382 participants, 495 participants with missing data of serum uric acid levels were also excluded. Thus, a total of 5,887 participants were selected for final analysis. Written informed consent was obtained from all participants before completing the survey. This study was approved by the Institutional Review Board (IRB) of Soonchunhyang University Hospital Bucheon (IRB no. 2018-05-017).

\section{Demographic variables and data collection}

The KNHANES was conducted by four special research teams. Each team was composed of eight experts including nurses, nutritionists, and students majoring in public health. The selected professional investigator was placed at the investigation site after completing one month of education and practice. Subsequently, the ability of the investigator to conduct research was verified through regular education and on-site quality management. A standardized interview was performed in participants' homes. An established questionnaire was used to collect information on demographic and socioeconomic characteristics, including age, sex, income, region, education, marital status, alcohol consumption, and smoking status. Alcohol consumption was categorized into the following four groups based on the frequency of alcohol consumption during the past year: never, $\leq 1$ time/week, $2 \sim$ 3 times/week, and $\geq 4$ times/week. Income levels were categorized into quartiles based on average individual monthly income. Urban and rural areas were classified by administrative district. Blood pressure was measured by nurses using standard methods. After at least five minutes of rest in a sitting position, blood pressure was measured using a mercury sphygmomanometer on the right arm. Blood pressure was measured three times. The mean of the second and third measurements was calculated and used for analysis. Blood samples were collected from each participant after at least eight hours of fasting. All blood samples were immediately refrigerated and transported in cold container to a central testing facility. Hypertension was defined as mean systolic blood pressure (SBP) $\geq 140 \mathrm{mmHg}$ or diastolic blood pressure (DBP) $\geq 90 \mathrm{mmHg}$ or by the presence of antihypertensive agents. Prehypertension was defined as an average SBP of $120 \sim 139 \mathrm{mmHg}$ or DBP of $80 \sim 89 \mathrm{mmHg}$. Diabetes mellitus was defined as a fasting plasma glucose $\geq 126$ $\mathrm{mg} / \mathrm{dL}$ or current use of antidiabetic drugs or insulin due to a previous diagnosis of diabetes. Impaired fasting glucose was determined if fasting plasma glucose level was $\geq 100 \mathrm{mg} / \mathrm{dL}$ and $<126 \mathrm{mg} / \mathrm{dL}$. Hypercholesterolemia was defined as a total plasma cholesterol $\geq 240 \mathrm{mg} / \mathrm{dL}$ after eight hours of fasting or current use of cholesterol-lowering agents. Hypertriglyceridemia was defined as triglyceride levels $>200 \mathrm{mg} / \mathrm{dL}$ after 12 hours of fasting. Metabolic syndrome was defined according to the modified National Cholesterol Education Program Adult Treatment Panel III (ATP III) definition [18]. Current ATP III criteria were used to define metabolic syndrome if 
any three of the following five traits were present: (1) central obesity (waist circumference $\geq 90 \mathrm{~cm}$ in Asian men or $\geq 80 \mathrm{~cm}$ in Asian women), (2) hypertriglyceridemia (fasting serum triglycerides $\geq 150 \mathrm{mg} / \mathrm{dL}$ or drug treat- ment for elevated triglycerides), (3) decreased high density lipoprotein (HDL) cholesterol (serum HDL cholesterol $<40 \mathrm{mg} / \mathrm{dL}$ in men or $<50 \mathrm{mg} / \mathrm{dL}$ in women or drug treatment for low HDL cholesterol), (4) elevated BP (SBP

Table 1. Clinical characteristics of participants

\begin{tabular}{|c|c|c|c|c|}
\hline Variables & $\begin{array}{c}\text { Total } \\
(\mathrm{n}=5,887)\end{array}$ & $\begin{array}{c}\text { Men } \\
(\mathrm{n}=2,568)\end{array}$ & $\begin{array}{l}\text { Women } \\
(\mathrm{n}=3,319)\end{array}$ & p-value \\
\hline Age (yr) & $46.7 \pm 0.3$ & $45.7 \pm 0.3$ & $47.7 \pm 0.4$ & $<0.001$ \\
\hline Sex & 6,129 & $2,647(49.75)$ & $3,482(50.25)$ & \\
\hline Income & & & & 0.688 \\
\hline Low & $1,464(25.8)$ & $638(26.1)$ & $826(25.4)$ & \\
\hline Mid-low & $1,468(24.4)$ & $638(24.0)$ & $830(25.0)$ & \\
\hline Mid-high & $1,476(25.0)$ & $640(24.7)$ & $836(25.3)$ & \\
\hline High & $1,458(24.8)$ & $641(25.2)$ & $817(24.3)$ & \\
\hline Education & & & & $<0.001$ \\
\hline Elementary school & $1,189(15.1)$ & $369(9.7)$ & $820(20.5)$ & \\
\hline Middle school & $576(9.1)$ & $252(8.7)$ & $324(9.5)$ & \\
\hline High school & 1,794 (36.2) & $808(37.0)$ & $986(35.4)$ & \\
\hline College graduation & 2,045 (39.6) & $998(44.6)$ & $1,047(34.6)$ & \\
\hline Marital status & & & & $<0.001$ \\
\hline Married & $4,970(77.3)$ & $2,081(72.4)$ & 2,889 (82.2) & \\
\hline Not married & $917(22.7)$ & 487 (27.6) & $430(17.8)$ & \\
\hline Region & & & & 0.660 \\
\hline Urban & $4,740(84.3)$ & $2,066(84.5)$ & $2,674(84.2)$ & \\
\hline Rural & $1,147(15.7)$ & $502(15.5)$ & 645 (15.8) & \\
\hline Alcohol consumption & & & & $<0.001$ \\
\hline Never & $928(15.1)$ & $318(10.0)$ & $610(20.8)$ & \\
\hline$\leq 1 /$ week & $2,889(58.1)$ & $1,168(52.0)$ & $1,721(64.9)$ & \\
\hline $2 \sim 3 /$ week & 877 (18.5) & $581(24.9)$ & $296(11.4)$ & \\
\hline$\geq 4 /$ week & $418(8.3)$ & $339(13.1)$ & $79(2.9)$ & \\
\hline Smoking & & & & $<0.001$ \\
\hline Never smoker & $3,490(56.2)$ & $579(24.7)$ & $2,911(87.9)$ & \\
\hline Ex-smoker & $1,204(20.9)$ & $1,028(35.9)$ & $176(5.8)$ & \\
\hline Current smoker & $1,098(22.9)$ & 919 (39.4) & $182(6.3)$ & \\
\hline Uric acid (mg/dL) & $5.09 \pm 0.02$ & $5.83 \pm 0.03$ & $4.36 \pm 0.02$ & $<0.001$ \\
\hline BMI $\left(\mathrm{kg} / \mathrm{m}^{2}\right)$ & $24.0 \pm 0.1$ & $24.5 \pm 0.1$ & $23.4 \pm 0.1$ & $<0.001$ \\
\hline Hypertension & $1,909(28.0)$ & $938(31.4)$ & $971(24.6)$ & $<0.001$ \\
\hline Diabetes & $744(10.8)$ & $373(11.6)$ & $371(10.0)$ & $<0.001$ \\
\hline Hypercholesterolemia & 1,209 (19.1) & $465(18.0)$ & $744(20.3)$ & 0.040 \\
\hline Hypertriglyceridemia & 677 (16.1) & $388(22.0)$ & $289(10.9)$ & $<0.001$ \\
\hline Stroke & $121(1.6)$ & $68(1.9)$ & $53(1.3)$ & 0.003 \\
\hline Myocardial infarction or angina & $165(2.2)$ & $94(2.7)$ & $71(1.7)$ & 0.012 \\
\hline Number of MetS components & & & & $<0.001$ \\
\hline 0 & 1,291 (21.9) & 448 (17.4) & $843(25.4)$ & \\
\hline 1 & $1,225(20.8)$ & $546(21.3)$ & $679(20.5)$ & \\
\hline 2 & $1,196(20.3)$ & $573(22.3)$ & $623(18.8)$ & \\
\hline 3 & 965 (16.4) & 496 (19.3) & $469(14.1)$ & \\
\hline 4 & 793 (13.5) & 348 (13.6) & 445 (13.4) & \\
\hline 5 & $417(7.1)$ & $157(6.1)$ & $260(7.8)$ & \\
\hline MetS & $2,175(36.9)$ & $1,001(39.0)$ & $1,174(35.4)$ & 0.004 \\
\hline
\end{tabular}

Values are presented as mean \pm standard deviationor unweighted $\mathrm{n}$ (weighted \%). BMI: body mass index, MetS: metabolic syndrome. 
Hyemin Jeong et al.

Table 2. Clinical characteristics of participants with or without hyperuricemia

\begin{tabular}{|c|c|c|c|c|c|c|}
\hline \multirow[b]{2}{*}{ Variables } & \multicolumn{3}{|c|}{ Men } & \multicolumn{3}{|c|}{ Women } \\
\hline & $\begin{array}{c}\text { Normal UA } \\
\quad(\cup A<7) \\
(\mathrm{n}=2,085)\end{array}$ & $\begin{array}{l}\text { Hyperuricemia } \\
\qquad(\cup A \geq 7) \\
(n=483)\end{array}$ & p-value & $\begin{array}{c}\text { Normal UA } \\
(\mathrm{UA}<6) \\
(\mathrm{n}=3,088)\end{array}$ & $\begin{array}{l}\text { Hyperuricemia } \\
\quad(\cup A \geq 6) \\
(n=231)\end{array}$ & p-value \\
\hline Age (yr) & $46.5 \pm 0.4$ & $42.6 \pm 0.8$ & $<0.001$ & $47.3 \pm 0.5$ & $55.1 \pm 1.5$ & $<0.001$ \\
\hline Income & & & 0.088 & & & 0.211 \\
\hline Low & $500(25.1)$ & $138(30.2)$ & & $755(25.2)$ & $71(30.1)$ & \\
\hline Mid-low & $529(24.8)$ & $109(20.6)$ & & $771(24.9)$ & $59(24.7)$ & \\
\hline Mid-high & $517(24.2)$ & $123(26.7)$ & & $778(25.2)$ & $58(26.7)$ & \\
\hline High & $531(25.9)$ & $110(22.5)$ & & $776(24.7)$ & $41(18.5)$ & \\
\hline Education & & & 0.043 & & & 0.003 \\
\hline Elementary school & $321(10.5)$ & $48(6.3)$ & & $737(19.8)$ & $83(30.4)$ & \\
\hline Middle school & $200(8.3)$ & $52(10.0)$ & & $303(9.7)$ & $21(7.2)$ & \\
\hline High school & $646(36.3)$ & $162(39.8)$ & & $914(35.2)$ & $72(37.1)$ & \\
\hline College graduation & $807(44.9)$ & $191(43.9)$ & & $1,001(35.3)$ & $46(25.3)$ & \\
\hline Marital status & & & 0.001 & & & 0.431 \\
\hline Married & $1,720(74.3)$ & $361(64.6)$ & & $2,684(82.0)$ & $205(84.7)$ & \\
\hline Unmarried & $365(25.7)$ & $122(35.4)$ & & $404(18.0)$ & $26(15.3)$ & \\
\hline Region & & & 0.374 & & & 0.040 \\
\hline Urban & $1,669(84.1)$ & $397(86.1)$ & & $2,497(84.5)$ & $177(79.2)$ & \\
\hline Rural & $416(15.9)$ & $86(13.9)$ & & $591(15.5)$ & $54(20.8)$ & \\
\hline Alcohol consumption & & & 0.671 & & & 0.122 \\
\hline Never & $261(9.8)$ & $57(10.3)$ & & $558(20.7)$ & $52(23.1)$ & \\
\hline$\leq 1 /$ week & $960(52.5)$ & $208(50.1)$ & & $1,622(65.2)$ & $99(60.8)$ & \\
\hline $2 \sim 3 /$ week & $463(25.0)$ & $118(24.6)$ & & $276(11.4)$ & $20(10.0)$ & \\
\hline$\geq 4 /$ week & $259(12.7)$ & $80(15.0)$ & & $70(2.7)$ & $9(6.1)$ & \\
\hline Smoking & & & 0.821 & & & 0.004 \\
\hline Never smoker & $475(24.4)$ & $104(25.8)$ & & $2,718(88.2)$ & $193(84.2)$ & \\
\hline Ex-smoker & $846(36.2)$ & $182(34.5)$ & & $167(5.9)$ & $9(3.8)$ & \\
\hline Current smoker & 724 (39.4) & 195 (39.7) & & $157(5.9)$ & $25(12.0)$ & \\
\hline Glucose (mg/dL) & $103.4 \pm 0.8$ & $100.6 \pm 0.8$ & 0.019 & $97.5 \pm 0.5$ & $104.3 \pm 1.8$ & 0.001 \\
\hline Hemoglobin A1c & $5.69 \pm 0.03$ & $5.58 \pm 0.03$ & 0.003 & $5.59 \pm 0.02$ & $5.86 \pm 0.06$ & $<0.001$ \\
\hline HDL-C (mg/dL) & $48.2 \pm 0.3$ & $44.6 \pm 0.6$ & $<0.001$ & $55.1 \pm 0.3$ & $49.9 \pm 0.9$ & $<0.001$ \\
\hline Triglyceride (mg/dL) & $160.5 \pm 4.5$ & $213.5 \pm 16.1$ & 0.002 & $114.8 \pm 1.9$ & $168.4 \pm 9.4$ & $<0.001$ \\
\hline WC $(\mathrm{cm})$ & $85.6 \pm 0.2$ & $89.4 \pm 0.5$ & $<0.001$ & $78.7 \pm 0.2$ & $87.1 \pm 0.9$ & $<0.001$ \\
\hline SBP (mmHg) & $120.0 \pm 0.4$ & $122.4 \pm 0.6$ & 0.002 & $114.8 \pm 0.4$ & $121.6 \pm 1.3$ & $<0.001$ \\
\hline $\mathrm{DBP}(\mathrm{mmHg})$ & $78.0 \pm 0.3$ & $80.6 \pm 0.5$ & $<0.001$ & $73.3 \pm 0.2$ & $74.6 \pm 0.8$ & 0.117 \\
\hline Number of MetS components & & & $<0.001$ & & & $<0.001$ \\
\hline 0 & $399(22.8)$ & $49(11.6)$ & & $831(30.3)$ & $12(6.2)$ & \\
\hline 1 & $469(22.8)$ & 77 (18.4) & & $655(23.1)$ & $24(14.4)$ & \\
\hline 2 & $464(21.9)$ & $109(21.1)$ & & $584(18.4)$ & $39(16.7)$ & \\
\hline 3 & 369 (15.9) & $127(25.9)$ & & $418(12.0)$ & $51(21.2)$ & \\
\hline 4 & $267(12.8)$ & $81(16.2)$ & & $394(11.0)$ & $51(19.9)$ & \\
\hline 5 & $117(4.8)$ & $40(6.8)$ & & $206(5.2)$ & $54(21.6)$ & \\
\hline MetS & $753(32.5)$ & $248(48.8)$ & $<0.001$ & $1,018(28.3)$ & $156(62.6)$ & $<0.001$ \\
\hline Classification & & & 0.001 & & & $<0.001$ \\
\hline Normal range & $1,110(60.3)$ & $256(59.5)$ & & $2,103(73.7)$ & $92(43.4)$ & \\
\hline IFG & $572(26.9)$ & $166(33.6)$ & & $564(17.2)$ & $83(34.4)$ & \\
\hline Diabetes mellitus & $325(12.8)$ & $48(6.9)$ & & $320(9.1)$ & $51(22.2)$ & \\
\hline Classification & & & 0.013 & & & $<0.001$ \\
\hline Normal range & $722(38.5)$ & $132(29.9)$ & & $1,634(57.7)$ & $63(31.2)$ & \\
\hline Prehypertension & $615(31.2)$ & $148(34.2)$ & & 603 (19.4) & 43 (20.4) & \\
\hline Hypertension & $735(30.3)$ & 203 (35.9) & & $846(22.9)$ & $125(48.4)$ & \\
\hline
\end{tabular}


Table 2. Continued

\begin{tabular}{|c|c|c|c|c|c|c|}
\hline \multirow[b]{2}{*}{ Variables } & \multicolumn{3}{|c|}{ Men } & \multicolumn{3}{|c|}{ Women } \\
\hline & $\begin{array}{c}\text { Normal UA } \\
\quad(\cup A<7) \\
(n=2,085)\end{array}$ & $\begin{array}{l}\text { Hyperuricemia } \\
\qquad(\cup A \geq 7) \\
(n=483)\end{array}$ & p-value & $\begin{array}{c}\text { Normal UA } \\
(\mathrm{UA}<6) \\
(\mathrm{n}=3,088)\end{array}$ & $\begin{array}{l}\text { Hyperuricemia } \\
\qquad \begin{array}{l}(\cup A \geq 6) \\
(n=231)\end{array}\end{array}$ & p-value \\
\hline Hypercholesterolemia & $380(18.2)$ & $85(17.1)$ & 0.658 & $673(19.5)$ & $71(31.7)$ & $<0.001$ \\
\hline Hypertriglyceridemia & $268(18.4)$ & $120(36.5)$ & $<0.001$ & $242(9.8)$ & $47(24.7)$ & $<0.001$ \\
\hline $\mathrm{BMI}\left(\mathrm{kg} / \mathrm{m}^{2}\right)$ & $24.26 \pm 0.09$ & $25.93 \pm 0.20$ & $<0.001$ & $23.22 \pm 0.09$ & $26.09 \pm 0.36$ & $<0.001$ \\
\hline Variable & & & $<0.001$ & & & $<0.001$ \\
\hline Underweight & $60(3.0)$ & $5(1.1)$ & & $154(5.8)$ & $3(1.2)$ & \\
\hline Normal range & $1,233(58.1)$ & $223(44.9)$ & & $2,020(67.2)$ & $86(38.6)$ & \\
\hline Overweight & 789 (38.9) & $254(54.1)$ & & $885(27.0)$ & $140(60.1)$ & \\
\hline Stroke & $59(2.1)$ & $9(1.3)$ & 0.288 & $46(1.3)$ & $7(2.0)$ & 0.407 \\
\hline Myocardial infarction or angina & $81(2.8)$ & $13(1.9)$ & 0.240 & $66(1.8)$ & $5(1.7)$ & 0.985 \\
\hline
\end{tabular}

Values are presented as mean \pm standard deviation or unweighted $\mathrm{n}$ (weighted \%). UA: uric acid, HDL-C: high-density lipoprotein cholesterol, WC: waist circumference, SBP: systolic blood pressure, DBP: diastolic blood pressure, MetS: metabolic syndrome, IFG: impaired fasting glucose, BMI: body mass index.

$\geq 130 \mathrm{mmHg}$ and/or DBP $\geq 85 \mathrm{mmHg}$ or drug treatment for elevated BP), and (5) hyperglycemia (fasting plasma glucose $\geq 100 \mathrm{mg} / \mathrm{dL}$ or drug treatment for elevated blood glucose).

Myocardial infarction or angina and stroke were defined in the questionnaire (question: was your myocardial infarction or angina diagnosed by a physician?) as "myocardial infarction or angina diagnosed by a physician" through a standardized interview. The questionnaire consisted of three responses (1, Yes; 2, No; 3, I have never been sick before). Participants who chose 1 (Yes) were classified as having hypertension. Each interview was conducted individually by a trained professional investigator. Height and weight were assessed using standardized techniques and equipment. Height was measured to the nearest 0.1 $\mathrm{cm}$ using a portable stadiometer Seca 225 (Seca, Hamburg, Germany). Weight was measured to the nearest $0.1 \mathrm{~kg}$ using a GL-6000-20 (G-tech, Uijeongbu, Korea). Body mass index (BMI) was calculated as weight divided by height squared $(\mathrm{kg} / \mathrm{m} 2)$. For waist circumference measurement, participants were asked to breathe out and the waist girth at the mid-point between the lower margin of ribs and the iliac crest was measured to the nearest 0.1 $\mathrm{cm}$ using a Seca 200 (Seca).

\section{Statistical analyses}

To reflect representative estimates of the noninstitutionalized Korean civilian population, survey sample weights were applied in all analyses. Sample weights were calculated by taking into account sampling rate, response rate, and age/sex proportions of the reference population (2005 Korean National Census registry). Clinical characteristics were analyzed according to dichotomized serum uric acid or quartiles of uric acid. Due to sex difference in uric acid level, the definition of hyperuricemia was different between men and women $(>7 \mathrm{mg} / \mathrm{dL}$ for men and $>6 \mathrm{mg} / \mathrm{dL}$ for women). To analyze the effect of uric acid on morbidity, univariable logistic regression models were computed with comorbidities as dependent variables and serum uric acid level as an independent variable. Multivariable logistic regression analyses were performed after adjusting for age, income, region, education, marriage, smoking, alcohol consumption, BMI, and estimated glomerular filtration rate (eGFR). The strength of association was estimated using odds ratio (OR) and $95 \%$ confidence intervals (95\% CIs). All statistical analyses were performed using SPSS statistics version 20.0 (IBM Corp., Armonk, NY, USA). All p-values were two-sided and a p-value of less than 0.05 was considered statistically significant.

\section{RESULTS}

Demographic characteristics of the study population are summarized in Table 1. Their mean age was 46.7 years. Males accounted for $49.7 \%$. Prevalence of hypertension, diabetes, hypertriglyceridemia, stroke, myocardial infarction or angina, and metabolic syndrome were significantly higher in men than in women. Serum uric acid was higher in men than in women. Clinical characteristics 
Hyemin Jeong et al.

Table 3. Clinical characteristics of women according to the menopause and uric acid

\begin{tabular}{|c|c|c|c|c|c|c|}
\hline \multirow{3}{*}{ Variables } & \multicolumn{6}{|c|}{ Women } \\
\hline & \multicolumn{3}{|c|}{ Premenopause $(n=1,715)$} & \multicolumn{3}{|c|}{ Menopause $(n=1,598)$} \\
\hline & $\begin{array}{c}\text { Normal UA } \\
(\cup A<6) \\
(n=1,638)\end{array}$ & $\begin{array}{l}\text { Hyperuricemia } \\
\qquad \begin{array}{c}(\cup A \geq 6) \\
(n=77)\end{array}\end{array}$ & p-value & $\begin{array}{c}\text { Normal UA } \\
(\cup A<6) \\
(n=1,444)\end{array}$ & $\begin{array}{l}\text { Hyperuricemia } \\
\qquad(\cup A \geq 6) \\
(n=154)\end{array}$ & $p$-value \\
\hline Age (yr) & $36.79 \pm 0.33$ & $36.81 \pm 1.80$ & 0.992 & $62.68 \pm 0.33$ & $66.76 \pm 0.98$ & $<0.001$ \\
\hline Income & & & 0.023 & & & 0.893 \\
\hline Low & $401(25.1)$ & $32(38.6)$ & & $352(25.0)$ & $39(24.7)$ & \\
\hline Mid-low & $408(25.0)$ & $17(19.6)$ & & $362(24.8)$ & $42(28.0)$ & \\
\hline Mid-high & $409(25.1)$ & $21(31.0)$ & & $367(25.3)$ & $37(23.8)$ & \\
\hline High & $415(24.8)$ & $7(10.8)$ & & $360(24.9)$ & $34(23.5)$ & \\
\hline Education & & & 0.932 & & & 0.063 \\
\hline Elementary school & $38(2.3)$ & $1(2.5)$ & & $698(43.9)$ & $82(45.9)$ & \\
\hline Middle school & $52(3.7)$ & $3(2.2)$ & & $251(18.0)$ & $18(9.9)$ & \\
\hline High school & $593(42.1)$ & $29(41.1)$ & & $320(25.6)$ & $43(34.9)$ & \\
\hline College graduation & $826(51.9)$ & $35(54.2)$ & & $172(12.5)$ & $11(9.3)$ & \\
\hline Marital status & & & 0.196 & & & 0.643 \\
\hline Married & $1,248(70.3)$ & $52(61.7)$ & & $1,430(99.1)$ & $153(99.4)$ & \\
\hline Unmarried & $390(29.7)$ & $25(38.3)$ & & $14(0.9)$ & $1(0.6)$ & \\
\hline Region & & & 0.032 & & & 0.808 \\
\hline Urban & $1,390(87.3)$ & $56(78.7)$ & & $1,102(80.4)$ & $121(79.5)$ & \\
\hline Rural & $248(12.7)$ & $21(21.3)$ & & $342(19.6)$ & $33(20.5)$ & \\
\hline Alcohol consumption & & & $<0.001$ & & & 0.589 \\
\hline Never & $256(16.4)$ & $12(12.6)$ & & $300(28.5)$ & $40(31.4)$ & \\
\hline$\leq 1 /$ week & $998(68.2)$ & $37(60.2)$ & & $622(59.8)$ & $62(61.1)$ & \\
\hline $2 \sim 3 /$ week & $201(13.1)$ & $12(15.0)$ & & $75(8.2)$ & $8(6.1)$ & \\
\hline$\geq 4 /$ week & $37(2.3)$ & $8(12.2)$ & & $33(3.5)$ & $1(1.4)$ & \\
\hline Smoking & & & $<0.001$ & & & 0.543 \\
\hline Never smoker & 1,383 (85.8) & $56(76.8)$ & & 1,331 (91.6) & $137(88.7)$ & \\
\hline Ex-smoker & $125(8.2)$ & $4(4.9)$ & & $41(2.6)$ & $5(3.1)$ & \\
\hline Current smoker & $96(6.0)$ & $13(18.3)$ & & $61(5.8)$ & $12(8.2)$ & \\
\hline Glucose (mg/dL) & $93.26 \pm 0.58$ & $95.5 \pm 1.82$ & 0.245 & $103.82 \pm 0.85$ & $109.99 \pm 0.38$ & 0.019 \\
\hline Hemoglobin A1c & $5.39 \pm 0.01$ & $5.42 \pm 0.07$ & 0.003 & $6.10 \pm 0.04$ & $6.12 \pm 0.07$ & $<0.001$ \\
\hline $\mathrm{HDL}-\mathrm{C}(\mathrm{mg} / \mathrm{dL})$ & $56.91 \pm 0.36$ & $52.56 \pm 1.95$ & 0.028 & $52.53 \pm 0.46$ & $48.34 \pm 1.02$ & $<0.001$ \\
\hline Triglyceride (mg/dL) & $100.65 \pm 1.98$ & $169.51 \pm 19.68$ & 0.001 & $135.86 \pm 3.36$ & $167.76 \pm 8.45$ & 0.001 \\
\hline WC $(\mathrm{cm})$ & $76.28 \pm 0.34$ & $85.24 \pm 1.74$ & $<0.001$ & $82.33 \pm 0.33$ & $88.29 \pm 0.98$ & $<0.001$ \\
\hline SBP (mmHg) & $108.79 \pm 0.37$ & $115.20 \pm 1.99$ & 0.001 & $123.88 \pm 0.59$ & $125.68 \pm 1.58$ & 0.001 \\
\hline $\mathrm{DBP}(\mathrm{mmHg})$ & $72.06 \pm 0.27$ & $77.17 \pm 1.54$ & 0.001 & $75.19 \pm 0.32$ & $73.08 \pm 0.91$ & 0.023 \\
\hline Number of MetS components & & & $<0.001$ & & & $<0.001$ \\
\hline 0 & $685(43.1)$ & $7(11.3)$ & & $146(11.5)$ & $5(3.0)$ & \\
\hline 1 & $430(27.0)$ & $16(25.4)$ & & $223(17.1)$ & $8(7.5)$ & \\
\hline 2 & $281(17.0)$ & $21(22.1)$ & & $301(20.4)$ & $18(13.3)$ & \\
\hline 3 & $129(6.9)$ & $9(11.5)$ & & $228(19.5)$ & $42(27.5)$ & \\
\hline 4 & $82(4.4)$ & $15(14.8)$ & & $301(20.8)$ & $36(23.1)$ & \\
\hline 5 & $31(1.6)$ & $9(14.9)$ & & $175(10.7)$ & $45(25.6)$ & \\
\hline MetS & $242(12.9)$ & $33(41.2)$ & $<0.001$ & $774(51.0)$ & $123(76.2)$ & $<0.001$ \\
\hline Classification & & & $<0.001$ & & & $<0.001$ \\
\hline Normal range & 1,304 (83.7) & $44(61.5)$ & & $796(59.0)$ & $48(31.9)$ & \\
\hline IFG & 224 (12.9) & $26(34.7)$ & & $338(23.3)$ & $57(34.2)$ & \\
\hline Diabetes mellitus & $63(3.4)$ & $5(3.8)$ & & $257(17.7)$ & $46(33.9)$ & \\
\hline
\end{tabular}


Table 3. Continued

\begin{tabular}{|c|c|c|c|c|c|c|}
\hline \multirow{3}{*}{ Variables } & \multicolumn{6}{|c|}{ Women } \\
\hline & \multicolumn{3}{|c|}{ Premenopause $(n=1,715)$} & \multicolumn{3}{|c|}{ Menopause $(n=1,598)$} \\
\hline & $\begin{array}{c}\text { Normal UA } \\
(\cup A<6) \\
(n=1,638)\end{array}$ & $\begin{array}{l}\text { Hyperuricemia } \\
\qquad \begin{array}{c}(\cup A \geq 6) \\
(n=77)\end{array}\end{array}$ & p-value & $\begin{array}{c}\text { Normal UA } \\
(\mathrm{UA}<6) \\
(\mathrm{n}=1,444)\end{array}$ & $\begin{array}{l}\text { Hyperuricemia } \\
\quad(\cup A \geq 6) \\
(n=154)\end{array}$ & $p$-value \\
\hline Classification & & & $<0.001$ & & & 0.002 \\
\hline Normal range & $1,226(75.9)$ & $34(46.8)$ & & $405(30.7)$ & $29(21.2)$ & \\
\hline Prehypertension & $259(16.3)$ & $23(29.3)$ & & $343(24.0)$ & $20(14.8)$ & \\
\hline Hypertension & $149(7.8)$ & $20(23.9)$ & & $696(45.3)$ & $105(64.0)$ & \\
\hline Hypercholesterolemia & $131(7.4)$ & $21(29.8)$ & $<0.001$ & $542(37.7)$ & $50(32.9)$ & 0.350 \\
\hline Hypertriglyceridemia & $78(6.4)$ & $13(20.6)$ & $<0.001$ & $164(14.7)$ & $34(27.3)$ & 0.001 \\
\hline $\mathrm{BMI}\left(\mathrm{kg} / \mathrm{m}^{2}\right)$ & $22.59 \pm 0.12$ & $26.05 \pm 0.64$ & $<0.001$ & $24.14 \pm 0.11$ & $26.12 \pm 0.39$ & $<0.001$ \\
\hline Variable & & & $<0.001$ & & & $<0.001$ \\
\hline Underweight & $122(8.2)$ & $2(1.9)$ & & $32(2.4)$ & $1(0.8)$ & \\
\hline Normal range & $1,134(70.9)$ & $27(39.2)$ & & $885(61.8)$ & $59(38.3)$ & \\
\hline Overweight & 359 (20.9) & $47(58.9)$ & & $526(35.8)$ & $93(60.9)$ & \\
\hline Stroke & $5(0.3)$ & $0(0)$ & 0.077 & $41(2.7)$ & $7(3.2)$ & 0.717 \\
\hline Myocardial infarction or angina & $4(0.2)$ & $0(0)$ & 0.706 & $62(3.9)$ & $5(2.7)$ & 0.438 \\
\hline
\end{tabular}

Values are presented as mean \pm standard deviation or unweighted $\mathrm{n}$ (weighted \%). UA: uric acid, HDL-C: high-density lipoprotein cholesterol, WC: waist circumference, SBP: systolic blood pressure, DBP: diastolic blood pressure, MetS: metabolic syndrome, IFG: impaired fasting glucose, BMI: body mass index.

according to the presence of hyperuricemia are shown in Table 2. The mean age of those in the hyperuricemia group was 42.6 years for men and 55.1 years for women. Participants with hyperuricemia showed higher prevalence of hypertension, metabolic syndrome, and hypertriglyceridemia than participants without hyperuricemia for both sexes. Diabetes was found more frequently in men with normal uric acid than in men with hyperuricemia. On the other hand, prevalence of diabetes was lower in women with normal uric acid than in women with hyperuricemia. Metabolic syndrome parameters were significantly higher in the hyperuricemia group than in the normal uric acid group for both sexes. Subgroup analysis was performed for women according to menopause (Table 3). Among 3,319 women, six participants who had no information about menopause were excluded. Among 3,316 women participants, 1,715 were premenopausal women and 1,598 were postmenopausal women. For postmenopausal women, the mean age was higher in the hyperuricemia group than in the normal uric acid group. However, the mean age was not significantly different between the hyperuricemia group and the normal uric acid group for premenopausal women. Hyperuricemia was associated with frequent use of alcohol and tobacco, particularly in premenopausal women. There was no as- sociation between hyperuricemia and alcohol consumption or smoking in postmenopausal women. In both premenopausal women and postmenopausal women, proportions of those with metabolic syndrome, hypertension, and obesity were higher in the hyperuricemia group than in the normal uric acid group. Proportion of diabetes was also significantly higher in the hyperuricemia group than in the normal uric acid group for both premenopausal women and premenopausal women, although the difference was greater in the postmenopausal group. For premenopausal women, proportions of those with diabetes in the normal uric acid group and the hyperuricemia group were $3.4 \%$ and $3.8 \%$, respectively. For postmenopausal women, proportions of diabetes in the normal uric acid group and the hyperuricemia group were $17.7 \%$ and $33.9 \%$, respectively.

Table 4 shows clinical comorbidities according to uric acid quartile. Prevalence of metabolic syndrome, hypertension, and BMI were significantly higher in the highest quartile compared to those in the lowest quartile for both sexes. Interestingly, the lowest uric acid quartile group showed the highest prevalence of diabetes in men. In contrast, the highest uric acid quartile group showed the highest prevalence of diabetes in women. Increasing uric acid concentration was associated with increasing preva- 


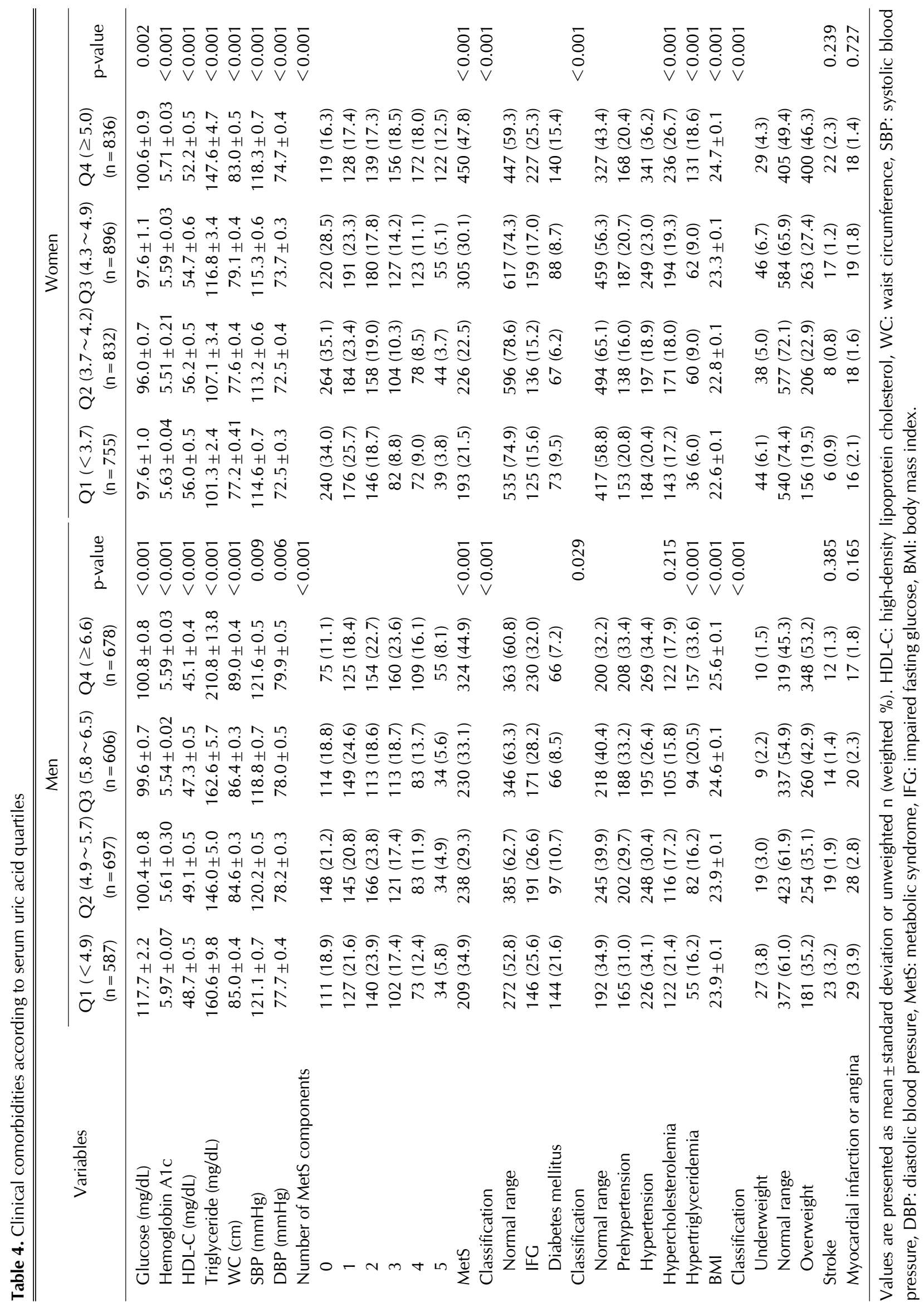




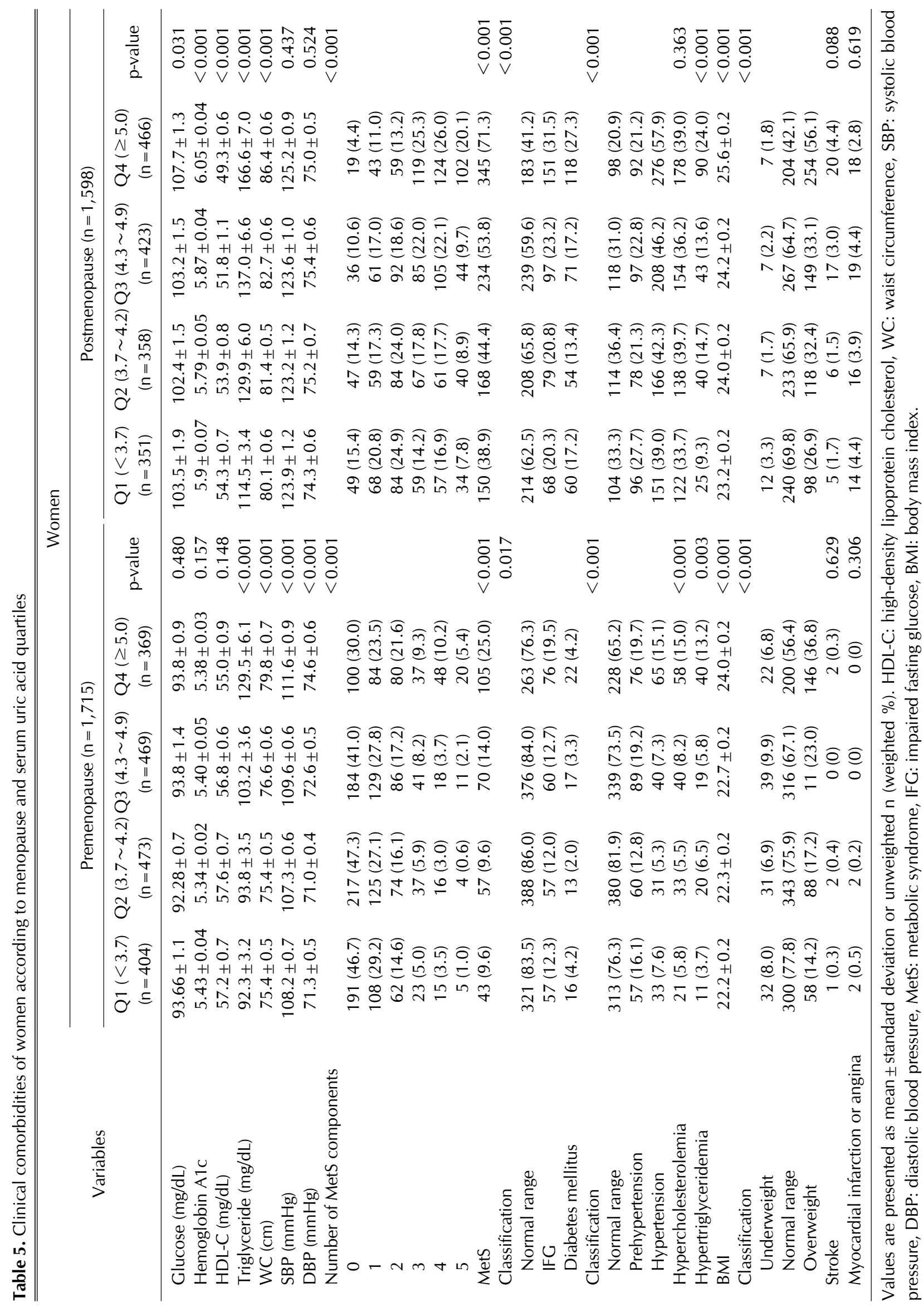




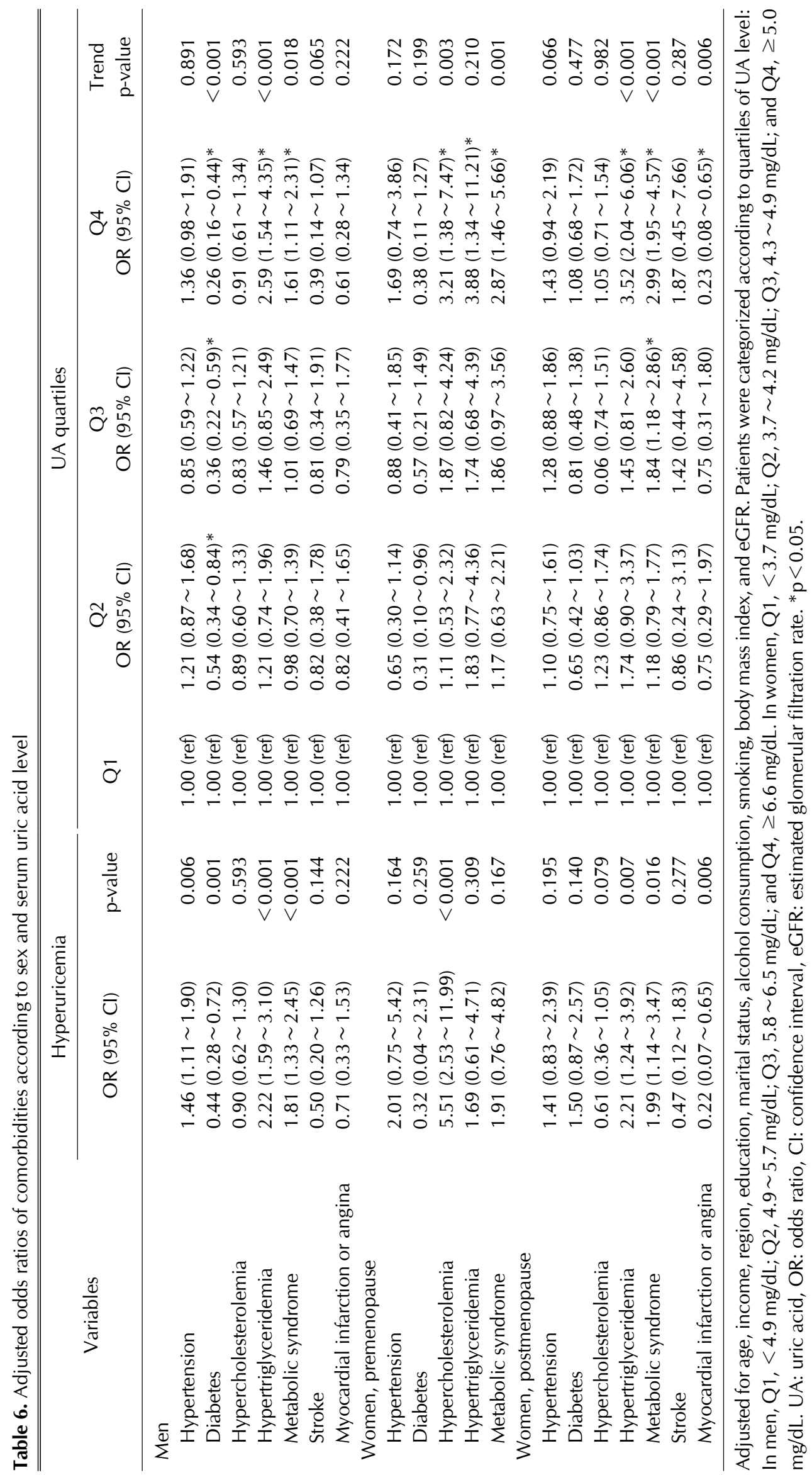


lence of metabolic syndrome, hypertension, and diabetes regardless of menopause (Table 5). In postmenopausal women, increasing uric acid concentration was significantly associated with increasing glucose and hemoglobin A1c levels, whereas there was no significant association between uric acid and glucose or hemoglobin A1c in the premenopausal group.

ORs and 95\% CIs of comorbidities after adjusting for age, socioeconomic characteristics, and eGFR in men are shown in Table 6. In men, hyperuricemia was significantly associated with a reduced prevalence of diabetes. Hyperuricemia was significantly associated with increased prevalence of hypertriglyceridemia. In women, hyperuricemia was significantly associated with increased prevalence of hypertriglyceridemia (OR: $2.12,95 \% \mathrm{CI}$ : 1.33 3.38, $\mathrm{p}=0.002$ ) and metabolic syndrome (OR: 1.95, 95\% CI: 1.22 3.13, $\mathrm{p}=0.006)$ after adjusting for age, socioeconomic characteristics, and eGFR. Hyperuricemia was negatively associated with the prevalence of myocardial infarction or angina (OR: 0.25, 95\% CI: 0.08 $0.75, p=0.013)$ in women. However, diabetes was not significantly associated with uric acid after adjustment (OR: 1.25, 95\% CI: $0.72 \sim 2.18, \mathrm{p}=0.418$ ) in women. When analysis was performed according to menopause, higher uric acid concentration was significantly associated with a reduced prevalence of myocardial infarction or angina in postmenopausal women. In premenopausal women, stroke, myocardial infarction, and angina could not be estimated due to a small number of patients. Increasing uric acid concentration was significantly associated with hypercholesterolemia in premenopausal women, whereas increasing uric acid concentration was significantly associated with hypertriglyceridemia in postmenopausal women. In both sexes, increasing uric acid concentration was significantly associated with increased prevalence of metabolic syndrome.

\section{DISCUSSION}

The association of serum uric acid and cardiovascular risk was investigated in the present study. Results showed that hyperuricemia was associated with an increased prevalence of metabolic syndrome in both sexes. Hyperuricemia was associated with a decreased prevalence of diabetes in men. Myocardial infarction or angina was negatively associated with uric acid in women. Associations of hyperuricemia with obesity, insulin resistance, and type 2 diabetes have been reported pre- viously [3]. It has been found that hyperuricemia contributes to insulin resistance by various mechanisms. Uric acid might cause direct oxidative stress on pancreatic islet cells and result in islet cell dysfunction [19]. It can also cause mitochondrial dysfunction in the liver by oxidative stress [20]. Uric acid can induce adipose tissue inflammation and result in decreased production of adiponectin [21]. However, studies on the association between type 2 diabetes and serum uric acid level have shown conflicting results. The current study found that serum uric acid was negatively associated with the prevalence of diabetes in men. These results correspond to those of a previous study reporting that subjects with the $3 \mathrm{rd}$ and the 4th quartiles of uric acid level had significantly lower prevalence of diabetes compared to those with the 1st quartile of uric acid level in Japanese men [14]. In Austrian men, serum uric acid was negatively correlated with fasting glucose level [22].

Inverse association between diabetes and hyperuricemia can be explained by uricosuric effect of glycosuria. Uricosuric effect of glycosuria occurs when blood glucose level is greater than approximately $10 \mathrm{mmol} / \mathrm{L}$ (180 $\mathrm{mg} / \mathrm{dL}$ ) [23]. Osmotic diuresis caused by glycosuria might enhance uric acid excretion. Uric acid is filtered in the glomerulus. Approximately $90 \%$ of uric acid is reabsorbed in the proximal renal tubule. Insulin resistance might enhance renal sodium retention, decrease urinary uric acid clearance, and increase serum uric acid concentration [24]. However, glucose competitively inhibits uric acid reabsorption at the same proximal tubule in diabetic individuals, resulting in decreased serum uric acid [25]. National Health and Nutritional Examination Survey (NHANES) III in US data showed that serum uric acid levels were increased with increasing HbAlc level up to the category of $6 \% \sim 6.9 \%$, but decreased with further increasing of HbAlc level, showing a bell-shaped relation [26]. Serum uric acid has been reported to be increased in the prediabetic stage but decreased in overt diabetes [27]. In the current study, a negative association between uric acid and diabetes was not found in women. In women, the prevalence of diabetes was higher in the hyperuricemia group than in the normouricemia group. After adjusting for age, income, region, education, marital status, alcohol consumption, smoking, BMI, and eGFR, there was no significant association between diabetes and uric acid concentration in women. This finding was in line with results of previous studies. Uric acid levels were associated with insulin resistance and plasma glucose levels more strong- 
ly in women than in men in a Taiwanese nondiabetic population [28]. A British primary care database study has shown that individuals with diabetes have a lower risk of gout independent of risk factors and that the inverse association between diabetes and gout is stronger in men than in women [29]. A population based cohort study in UK has shown that individuals with type 2 diabetes are at increased risk of gout and that this association is stronger in women. However, after adjusting for renal function and comorbidities, the risk disappeared in women and reversed in men [30]. The NHANES III study has reported that individuals with moderately elevated HbAlc levels (prediabetes) might be at an increased risk of hyperuricemia and gout in women, whereas those with highly elevated HbA1c levels (diabetes) might be at a lower risk of hyperuricemia and gout in men [26].

Reasons for these sex-specific differences are unclear. Sex hormone might impact uric acid metabolism [31]. Serum uric acid levels in women are lower than in men. However, uric acid levels increase around menopause. Thus, the relative physiologic impact of hyperuricemia on women might be stronger than on men [32]. A possible hypothesis for this sex difference is that the effect of glucose on uric acid reabsorption in the kidney tubule is different between men and women [30]. We performed a subgroup analysis for women according to menopause. The prevalence of diabetes was higher in the hyperuricemia group than in the normal uric acid group for both premenopausal and postmenopausal women. However, after adjusting for age, income, region, education, marital status, alcohol consumption, smoking, BMI, and eGFR, there was no significant association between diabetes and uric acid concentration in premenopausal women or menopausal women. Sex difference in the association between uric acid and diabetes cannot be fully explained by sex hormone alone. The effect of sex on the association between uric acid and diabetes clearly needs further exploration.

We found that risks of a number of metabolic syndrome components increased with increasing levels of serum uric acid, in agreement with a previous study showing that serum uric acid was positively associated with metabolic syndrome in a Chinese community population [33]. A nationwide prospective follow-up study in Taiwan has found that hyperuricemia is a significant independent risk determinant for metabolic syndrome [34]. Currently available evidence suggests that uric acid might play a role in the development of metabolic syndrome. Since serum insulin reduces renal excretion of uric acid, elevated uric acid might be a consequence of hyperinsulinemia in metabolic syndrome [35]. However, hyperuricemia has been detected prior to the development of hyperinsulinemia [36]. Hyperuricemia-induced endothelial dysfunction and direct oxidative changes in adipocytes might be mechanisms underlying the association between hyperuricemia and metabolic syndrome [37].

The current study showed that elevated uric acid concentration was associated with a decreased prevalence of myocardial infarction or angina in women participants. Previous studies have reported that uric acid and cardiovascular disease have a J-shaped relationship [38]. Extremely low levels of uric acid have been associated with endothelial dysfunction, resulting in increased cardiovascular events and all-cause deaths. Low uric acid levels have been associated with adverse clinical outcomes in patients with vasospastic angina in a Korean population [39]. Patients with Prinzmetal's angina are predominantly younger women who might not have classical cardiovascular risk factors compared to those with classic atherosclerotic myocardial infarction or angina. Although pathophysiology of variant angina is not fully understood yet, hypouricemia-induced endothelial dysfunction might result in a U-shaped relationship between uric acid and outcomes of variant angina. The protective effect of uric acid on myocardial infarction or angina in women may be partially explained by pure variant angina or mixed angina patients. However, those with the highest quartile of uric acid level showed significantly increased risk of hypertension in participants of the present study compared to those with the lowest quartile of uric acid level. Moreover, the current study showed that hyperuricemia was associated with dyslipidemia and metabolic syndrome as other risk factors for coronary artery disease in women. Previous studies have reported that women with hyperuricemia show higher incidence of atherosclerosis and cardiovascular mortality than men [40]. In the current study, the definition of myocardial infarction or angina was based on self-report whereas definitions of diabetes, metabolic syndrome, and hypertension were based on laboratory results. Additionally, the total number of premenopausal women with myocardial infarction or angina was small. Moreover, multivariable analysis was not performed for premenopausal women. Although uric acid was negatively associated with myocardial infarction or angina in postmenopausal women, these results should be cautiously applied considering these 
factors.

This study had some limitations. First, the definition of myocardial infarction or angina and stroke depended on self-reported information provided by participants in an interview. Although trained researchers asked participants in face-to-face interviews, recall bias might have influenced self-reported data. Second, we could not assess medications for gout or cardiovascular diseases. Therefore, we were unable to evaluate the potential effect of medications on comorbidities or serum uric acid. Third, we could not determine causality due to the cross-sectional design of this study. The strength of this study was that we analyzed association of uric acid with cardiovascular risk factors using a nationwide representative sample of the Korean adult population after adjusting for socioeconomic and lifestyle characteristics. Our comprehensive cross-sectional data could provide additional evidence for the relationship between uric acid and cardiovascular risk factors.

\section{CONCLUSION}

Results of this study revealed that participants with hyperuricemia showed increased prevalence of metabolic syndrome. Hyperuricemia was associated with decreased prevalence of diabetes mellitus in men and decreased prevalence of myocardial infarction or angina in women. Thus, clinicians should be aware of comorbidities in patients with hyperuricemia and take appropriate preventive and management measures.

\section{CONFLICT OF INTEREST}

No potential conflict of interest relevant to this article was reported.

\section{AUTHOR CONTRIBUTIONS}

H.J. designed this study, data collection, performed statistical analysis, and drafted the manuscript. J.E.M. collect the data, performed statistical analysis. C.H.J. designed this study and critically reviewed the manuscript.

\section{REFERENCES}

1. Kim SY, Guevara JP, Kim KM, Choi HK, Heitjan DF, Albert DA. Hyperuricemia and coronary heart disease: a systematic review and meta-analysis. Arthritis Care Res (Hoboken)
2010;62:170-80.

2. Ford ES, Li C, Cook S, Choi HK. Serum concentrations of uric acid and the metabolic syndrome among US children and adolescents. Circulation 2007;115:2526-32.

3. Dehghan A, van Hoek M, Sijbrands EJ, Hofman A, Witteman JC. High serum uric acid as a novel risk factor for type 2 diabetes. Diabetes Care 2008;31:361-2.

4. Rho YH, Choi SJ, Lee YH, Ji JD, Choi KM, Baik SH, et al. Prevalence of the metabolic syndrome in patients with gout. J Korean Rheum Assoc 2004;11:349-57.

5. Hong Q, Qi K, Feng Z, Huang Z, Cui S, Wang L, et al. Hyperuricemia induces endothelial dysfunction via mitochondrial $\mathrm{Na}^{+} / \mathrm{Ca}^{+}$exchanger-mediated mitochondrial calcium overload. Cell Calcium 2012;51:402-10.

6. Lyngdoh T, Marques-Vidal P, Paccaud F, Preisig M, Waeber $\mathrm{G}$, Bochud M, et al. Elevated serum uric acid is associated with high circulating inflammatory cytokines in the population-based Colaus study. PLoS One 2011;6:e19901.

7. Strazzullo P, Puig JG. Uric acid and oxidative stress: relative impact on cardiovascular risk? Nutr Metab Cardiovasc Dis 2007; 17:409-14.

8. Johnson RJ, Nakagawa T, Sanchez-Lozada LG, Shafiu M, Sundaram S, Le M, et al. Sugar, uric acid, and the etiology of diabetes and obesity. Diabetes 2013;62:3307-15.

9. Cutler RG. Urate and ascorbate: their possible roles as antioxidants in determining longevity of mammalian species. Arch Gerontol Geriatr 1984;3:321-48.

10. Ames BN, Cathcart R, Schwiers E, Hochstein P. Uric acid provides an antioxidant defense in humans against oxidantand radical-caused aging and cancer: a hypothesis. Proc Natl Acad Sci U S A 1981;78:6858-62.

11. Iso T, Kurabayashi M. Extremely low levels of serum uric acid are associated with endothelial dysfunction in humans. Circ J 2015;79:978-80.

12. Waring WS, McKnight JA, Webb DJ, Maxwell SR. Uric acid restores endothelial function in patients with type 1 diabetes and regular smokers. Diabetes 2006;55:3127-32.

13. Bandaru P, Shankar A. Association between serum uric acid levels and diabetes mellitus. Int J Endocrinol 2011;2011: 604715.

14. Oda E, Kawai R, Sukumaran V, Watanabe K. Uric acid is positively associated with metabolic syndrome but negatively associated with diabetes in Japanese men. Intern Med 2009;48:1785-91.

15. Barbieri L, Verdoia M, Schaffer A, Marino P, Suryapranata H, De Luca G. Impact of sex on uric acid levels and its relationship with the extent of coronary artery disease: a single-centre study. Atherosclerosis 2015;241:241-8.

16. Kweon S, Kim Y, Jang MJ, Kim Y, Kim K, Choi S, et al. Data resource profile: the Korea National Health and Nutrition Examination Survey (KNHANES). Int J Epidemiol 2014;43: 69-77.

17. Jeong H, Baek SY, Kim SW, Park EJ, Lee J, Kim H, et al. C reactive protein level as a marker for dyslipidaemia, diabetes and metabolic syndrome: results from the Korea National Health and Nutrition Examination Survey. BMJ Open 2019;9:e029861.

18. Grundy SM, Cleeman JI, Daniels SR, Donato KA, Eckel RH, Franklin BA, et al. Diagnosis and management of the metabolic syndrome: an American Heart Association/National Heart, Lung, and Blood Institute Scientific Statement. 
Circulation 2005;112:2735-52.

19. Roncal-Jimenez CA, Lanaspa MA, Rivard CJ, Nakagawa T, Sanchez-Lozada LG, Jalal D, et al. Sucrose induces fatty liver and pancreatic inflammation in male breeder rats independent of excess energy intake. Metabolism 2011;60: 1259-70.

20. Lanaspa MA, Sanchez-Lozada LG, Choi YJ, Cicerchi C, Kanbay M, Roncal-Jimenez CA, et al. Uric acid induces hepatic steatosis by generation of mitochondrial oxidative stress: potential role in fructose-dependent and -independent fatty liver. J Biol Chem 2012;287:40732-44.

21. Baldwin W, McRae S, Marek G, Wymer D, Pannu V, Baylis $\mathrm{C}$, et al. Hyperuricemia as a mediator of the proinflammatory endocrine imbalance in the adipose tissue in a murine model of the metabolic syndrome. Diabetes 2011;60:1258-69.

22. Strasak A, Ruttmann E, Brant L, Kelleher C, Klenk J, Concin $\mathrm{H}$, et al.; VHM\&PP Study Group. Serum uric acid and risk of cardiovascular mortality: a prospective long-term study of 83,683 Austrian men. Clin Chem 2008;54:273-84.

23. Cook DG, Shaper AG, Thelle DS, Whitehead TP. Serum uric acid, serum glucose and diabetes: relationships in a population study. Postgrad Med J 1986;62:1001-6.

24. Reaven GM. The kidney: an unwilling accomplice in syndrome X. Am J Kidney Dis 1997;30:928-31.

25. Tuomilehto J, Zimmet P, Wolf E, Taylor R, Ram P, King H. Plasma uric acid level and its association with diabetes mellitus and some biologic parameters in a biracial population of Fiji. Am J Epidemiol 1988;127:321-36.

26. Choi HK, Ford ES. Haemoglobin A1c, fasting glucose, serum C-peptide and insulin resistance in relation to serum uric acid levels--the Third National Health and Nutrition Examination Survey. Rheumatology (Oxford) 2008;47:713-7.

27. Erdberg A, Boner G, van Dyk DJ, Carel R. Urine uric acid excretion in patients with insulin-dependent diabetes mellitus. Nephron 1992;60:134-7.

28. Chou P, Lin KC, Lin HY, Tsai ST. Gender differences in the relationships of serum uric acid with fasting serum insulin and plasma glucose in patients without diabetes. J Rheumatol 2001;28:571-6.

29. Rodríguez G, Soriano LC, Choi HK. Impact of diabetes against the future risk of developing gout. Ann Rheum Dis 2010;69:2090-4.
30. Wijnands JM, van Durme CM, Driessen JH, Boonen A, Klop $\mathrm{C}$, Leufkens B, et al. Individuals with type 2 diabetes mellitus are at an increased risk of gout but this is not due to diabetes: a population-based cohort study. Medicine (Baltimore) 2015;94:e1358.

31. Wingrove CS, Walton C, Stevenson JC. The effect of menopause on serum uric acid levels in non-obese healthy women. Metabolism 1998;47:435-8.

32. De Vera MA, Rahman MM, Bhole V, Kopec JA, Choi HK. Independent impact of gout on the risk of acute myocardial infarction among elderly women: a population-based study. Ann Rheum Dis 2010;69:1162-4.

33. Liu M, He Y, Jiang B, Wu L, Yang S, Wang Y, et al. Association between serum uric acid level and metabolic syndrome and its sex difference in a Chinese community elderly population. Int J Endocrinol 2014;2014:754678.

34. Yang T, Chu CH, Bai CH, You SL, Chou YC, Chou WY, et al. Uric acid level as a risk marker for metabolic syndrome: a Chinese cohort study. Atherosclerosis 2012;220:525-31.

35. Quiñones Galvan A, Natali A, Baldi S, Frascerra S, Sanna G, Ciociaro D, et al. Effect of insulin on uric acid excretion in humans. Am J Physiol 1995;268 (1 Pt 1):E1-5.

36. Carnethon MR, Fortmann SP, Palaniappan L, Duncan BB, Schmidt MI, Chambless LE. Risk factors for progression to incident hyperinsulinemia: the Atherosclerosis Risk in Communities Study, 1987-1998. Am J Epidemiol 2003;158: 1058-67.

37. Feig DI, Kang DH, Johnson RJ. Uric acid and cardiovascular risk. N Engl J Med 2008;359:1811-21.

38. Verdecchia P, Schillaci G, Reboldi G, Santeusanio F, Porcellati C, Brunetti P. Relation between serum uric acid and risk of cardiovascular disease in essential hypertension. The PIUMA study. Hypertension 2000;36:1072-8.

39. Gwag HB, Yang JH, Park TK, Song YB, Hahn JY, Choi JH, et al. Uric acid level has a u-shaped association with clinical outcomes in patients with vasospastic angina. J Korean Med Sci 2017;32:1275-80.

40. Fang J, Alderman MH. Serum uric acid and cardiovascular mortality the NHANES I epidemiologic follow-up study, 1971-1992. National Health and Nutrition Examination Survey. JAMA 2000;283:2404-10. 